\title{
Effective $Q-Q$ interactions in constituent quark models
}

\author{
L. Ya. Glozman, ${ }^{1}$ Z. Papp,${ }^{2}$ W. Plessas,${ }^{1}$ K. Varga,${ }^{2,3}$ and R. F. Wagenbrunn ${ }^{1}$ \\ ${ }^{1}$ Institute for Theoretical Physics, University of Graz, A-8010 Graz, Austria \\ ${ }^{2}$ Institute of Nuclear Research, Hungarian Academy of Sciences, H-4001 Debrecen, Hungary \\ ${ }^{3}$ Department of Physics, Niigata University, Niigata 950-21, Japan
}

\begin{abstract}
We study the performance of some recent potential models suggested as effective interactions between constituent quarks. In particular, we address constituent quark models for baryons with hybrid $Q-Q$ interactions stemming from one-gluon plus meson exchanges. Upon recalculating two of such models we find them to fail in describing the $N$ and $\Delta$ spectra. Our calculations are based on accurate solutions of the three-quark systems in both a variational Schrödinger and a rigorous Faddeev approach. It is argued that hybrid $Q-Q$ interactions encounter difficulties in describing baryon spectra due to the specific contributions from one-gluon and pion exchanges together. In contrast, a chiral constituent quark model with a $Q-Q$ interaction solely derived from Goldstone-boson exchange is capable of providing a unified description of both the $N$ and $\Delta$ spectra in good agreement with phenomenology.
\end{abstract}

12.39.-x, 14.20.-c, 21.45.+v

Typeset using REVTEX 


\section{INTRODUCTION}

Traditional constituent quark models (CQM) adopted one-gluon exchange (OGE) [1] as the interaction between constituent quarks $(Q)$. Over the years it has become evident that CQM relying solely on OGE $Q-Q$ interactions face some intriguing problems in light-baryon spectroscopy [2,3]. Most severe are:

(i) the wrong level orderings of positive- and negative-parity excitations in the $N, \Delta, \Lambda$, and $\Sigma$ spectra;

(ii) the missing flavour dependence of the $Q-Q$ interaction necessary, e.g., for a simultaneous description of the correct level orderings in the $N$ and $\Lambda$ spectra; and

(iii) the strong spin-orbit splittings that are produced by the OGE interaction but not found in the empirical spectra.

All of these effects can be traced back to inadequate symmetry properties inherent in the OGE interaction.

Beyond CQM with OGE dynamics only, hybrid models advocating in addition mesonexchange $Q-Q$ interactions have been suggested, see, e.g., Ref. [1]. With respect to the $N$ and $\Delta$ spectra, especially $\pi$ and $\sigma$ exchanges have been introduced to supplement the interaction between constituent quarks. Sometimes hybrid CQM have also been employed in constructing baryon-baryon interaction models, see, e.g., Refs. [由], 5].

Recently two groups, viz. Valcarce, González, Fernández, and Vento (VGFV) [6] and Dziembowski, Fabre, and Miller (DFM) [7] came up with new versions of hybrid constituent quark models. They presented $N$ and $\Delta$ excitation spectra and claimed to have achieved a reasonable consistent description thereof. In both works a sizeable contribution from the OGE interaction is maintained. In either case the hyperspherical-harmonics method (HHM) [8.9] with several simplifying assumptions was used to solve the nonrelativistic three- $Q$ problem. We have studied the models of VGFV and DFM in a detailed manner. In particular, we have checked the pertinent results for the $N$ and $\Delta$ spectra in two very reliable ways, namely, by calculating the three- $Q$ systems through 
(i) a precise solution of the Faddeev equations (via high-rank separable expansions) [10] and (ii) a stochastic variational solution of the Schrödinger equation [11].

Both of these methods have been extensively tested before not only for three- $Q$ systems like, e.g., for the CQM models of Refs. [12 [14 but also for nuclear and atomic three-body problems. Existing benchmark results in the literature have been met accurately, in particular for spin-isospin/flavour dependent forces, and even a wider class of two-body potentials [10,11]; for instance, the stochastic variational method has recently passed successful tests in three- $N$ (and also four- $N$ ) bound-state calculations with realistic $N-N$ potentials containing noncentral components of various spin-spin and tensor types [15]. While in all cases our results for the light-baryon spectra coincide with each other for both of the above methods (i) and (ii), they are found in striking disagreement with the results given in the papers of VGFV [6] and DFM [7]. For example, the $N-\Delta$ splitting is found to be about $600 \mathrm{MeV}$ with the DFM interaction and even bigger than $2000 \mathrm{MeV}$ for the VGFV model!

In the following section we give a detailed analysis of the VGFV and DFM models and outline their shortcomings. We present the corrected spectra and explain in which respects the physical implications drawn from erroneous results in the works of VGFV and DFM cannot be relied on. In Sec. III we discuss a constituent quark model whose effective $Q-Q$ interaction is derived from GBE alone [2]. In the $N$ and $\Delta$ cases the interaction is thus mediated by $\pi_{-}, \eta_{-}$, and $\eta^{\prime}$-exchanges [14]. Along a modified parametrization of the corresponding pseudoscalar meson-exchange potentials in a semirelativistic framework it is demonstrated that no OGE (or at most a very weak one) is needed to provide a unified description of the $N$ and $\Delta$ spectra in close agreement with phenomenology.

\section{CONSTITUENT QUARK MODELS WITH HYBRID $Q-Q$ INTERACTIONS}

Hybrid CQM advocate sizeable contributions from OGE and employ in addition meson exchanges for the effective interaction between constituent quarks. 


\section{A. The model by Valcarce, González, Fernández, and Vento}

In the model of VGFV [6] the following $Q-Q$ potential is used:

$$
V^{V G F V}=V_{O G E}+V_{O P E}+V_{O S E}+V_{\text {conf }} .
$$

The OGE potential is employed without tensor and spin-orbit parts in the actual calculations [16], i.e. in the form

$$
V_{O G E}\left(\vec{r}_{i j}\right)=\frac{1}{4} \alpha_{s} \vec{\lambda}_{i}^{C} \cdot \vec{\lambda}_{j}^{C}\left[\frac{1}{r_{i j}}-\frac{1}{4 m_{Q}^{2}}\left(1+\frac{2}{3} \vec{\sigma}_{i} \cdot \vec{\sigma}_{j}\right) \frac{e^{-r_{i j} / r_{0}}}{r_{i j} r_{0}^{2}}\right],
$$

where $\vec{\sigma}_{i}$ and $\vec{\lambda}_{i}^{C}$ are the quark spin and colour matrices, $r_{i j}$ is the interquark distance, and $m_{Q}$ the constituent quark mass; VGFV use $m_{Q}=313 \mathrm{MeV} . \alpha_{s}$ is the effective quarkgluon coupling constant and $r_{0}$ a parameter involved in the smearing of the $\delta$-function. The one-pion-exchange (OPE) potential $V_{O P E}$ is used in the form

$$
\begin{aligned}
V_{O P E}\left(\vec{r}_{i j}\right) & =\frac{g_{\pi Q}^{2}}{4 \pi} \frac{1}{12 m_{Q}^{2}} \overrightarrow{\tau_{i}} \cdot \overrightarrow{\tau_{j}} \frac{\Lambda^{2}}{\Lambda^{2}-\mu_{\pi}^{2}}\left\{\left(\mu_{\pi}^{2} \frac{e^{-\mu_{\pi} r_{i j}}}{r_{i j}}-\Lambda^{2} \frac{e^{-\Lambda r_{i j}}}{r_{i j}}\right) \overrightarrow{\sigma_{i}} \cdot \overrightarrow{\sigma_{j}}\right. \\
& \left.+\left[\left(1+\frac{3}{\mu_{\pi} r_{i j}}+\frac{3}{\mu_{\pi}^{2} r_{i j}^{2}}\right) \mu_{\pi}^{2} \frac{e^{-\mu_{\pi} r_{i j}}}{r_{i j}}-\left(1+\frac{3}{\Lambda r_{i j}}+\frac{3}{\Lambda^{2} r_{i j}^{2}}\right) \Lambda^{2} \frac{e^{-\Lambda r_{i j}}}{r_{i j}}\right] \hat{S}_{i j}\right\},
\end{aligned}
$$

where $g_{\pi Q}^{2} / 4 \pi$ is the effective pion-quark coupling constant, $\mu_{\pi}=0.7 \mathrm{fm}^{-1}$ the pion mass, and $\Lambda$ a parameter related to the extended $\pi Q$ vertex. $\hat{S}_{i j}$ is the tensor operator $\hat{S}_{i j}=$ $3\left(\overrightarrow{\sigma_{i}} \cdot \hat{r}_{i j}\right)\left(\overrightarrow{\sigma_{j}} \cdot \hat{r}_{i j}\right)-\overrightarrow{\sigma_{i}} \cdot \overrightarrow{\sigma_{j}}$. The one-sigma-exchange (OSE) potential $V_{O S E}$ takes simply the form

$$
V_{O S E}\left(\vec{r}_{i j}\right)=-\frac{g_{\pi Q}^{2}}{4 \pi} \frac{\Lambda^{2}}{\Lambda^{2}-\mu_{\sigma}^{2}}\left(\frac{e^{-\mu_{\sigma} r_{i j}}}{r_{i j}}-\frac{e^{-\Lambda r_{i j}}}{r_{i j}}\right)
$$

with the $\sigma$-mass $\mu_{\sigma}=3.42 \mathrm{fm}^{-1}$. Finally the confinement potential is assumed in linear form

$$
V_{\text {conf }}\left(\vec{r}_{i j}\right)=V_{0}+C r_{i j}
$$

with the strength $C=0.980 \mathrm{fm}^{-2}$ and a constant $V_{0}$ necessary to adjust the nucleon ground state level to its phenomenological value. The whole $Q-Q$ potential (1) involves 6 fitting 
parameters $\left(\alpha_{s}, g_{\pi Q}, r_{0}, \Lambda, C, V_{0}\right)$ if one considers the constituent-quark and meson masses

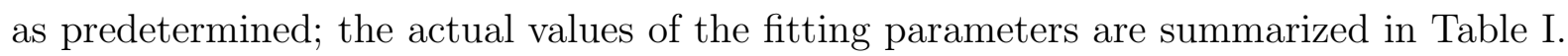

VGFV solved the three- $Q$ system along the Schrödinger equation with the HHM [8,9]. However, only a very restricted basis set was used for the expansion of the three- $Q$ wave functions (two terms $K=0,2$ for positive-parity states and one term $K=1$ for negativeparity states). VGFV published the results shown in Fig. 1a.

We have recalculated the $N$ and $\Delta$ spectra with the VGFV potential in Eq. (1) along both of our methods, i.e., (i) the precise solution of the Faddeev equations (via high-rank separable expansions) and (ii) the stochastic variational solution of the Schrödinger equation (as explained in the previous section; for details see also Refs. [10,11]). The results from both calculations agree perfectly with each other and they are shown in Fig. 1b.

¿From the comparison of the spectra in Figs. 1a and b, which have both been normalized to reproduce the nucleon ground state at $939 \mathrm{MeV}$ (in case of our calculations a numerical value of $V_{0}=+488 \mathrm{MeV}$ is to be employed for this purpose in the confinement potential (5)), several differences are evident. Most striking is the unrealistic $N-\Delta$ splitting, which turns out to be larger than $2000 \mathrm{MeV}$; notice that the $\Delta$ ground state has a mass of $\approx 3200$ $\mathrm{MeV}$ ! Also some of the $N^{*}$ levels, especially in the negative-parity $\frac{1}{2}^{-}, \frac{3}{2}^{-}, \frac{5}{2}^{-}$bands, show excitations above the $N$ ground state by far too large. Furthermore, additional levels (not given in Ref. [6]) appear. For instance, in the $\frac{1}{2}^{-}$band we reproduce the lowest state, which is predominantly $S=\frac{1}{2}, L=1$, at a mass of $\approx 1550 \mathrm{MeV}$. The next excitations, whose spin contents are still $S=\frac{1}{2}, L=1$ (with probabilities $\approx 99 \%$ ), are found between 2000 and $2500 \mathrm{MeV}$. Only much further above, at $\approx 3500 \mathrm{MeV}$, the $S=\frac{3}{2}, L=1$ excitation (marked by a dagger in Fig. 1b) appears. This is the state to be compared with the second $\frac{1}{2}^{-}$ excitation (also marked by a dagger) in the spectrum given by VGFV in Fig. 1a. Exactly the same structure is recovered in the $\frac{3}{2}^{-}$and $\frac{5}{2}^{-}$bands, with even an additional $S=\frac{1}{2}$, $L=3$ state appearing in the latter. In Ref. [6] also the prediction for the Roper resonance $N(1440)$ is given with a wrong energy. In fact, it lies above $1600 \mathrm{MeV}$ and thus also above the first negative-parity excitation (see Fig. 1b). Consequently the problem with the wrong 
level orderings of positive- and negative-parity states persists, contrary to the claim made by VGFV.

We conclude that the HHM as used by VGFV is inadequate to reproduce the correct spectra in the case of the $Q-Q$ potential (11)-(5) with the parameters chosen in Ref. [6]. The most important reason is that for certain spin-isospin channels, specifically for $S_{i j}=T_{i j}=0$, the potentials (2) and (3) become very deep at rather short distances $r_{i j}$. This is especially due to the OGE potential whose $\delta$-function term in the central and spin-spin parts involves an extremely small parameter $r_{0}$ (see Table 四). If also the OGE tensor part as given in formula (2) of Ref. [6] was employed, the three- $Q$ spectrum would not be bounded from below. Even in the case one uses just the OGE potential as in Eq. (2) above (with such a small $r_{0}$ as employed by VGFV) great care must be exerted in the expansion of test functions. While the actual calculation is not so dangerous in the $\Delta$ case (here, $S_{i j}=T_{i j}=1$, thus the OGE is repulsive and the OPE is attractive at short ranges), the $N$ case is rather tricky to compute (here, $S_{i j}=T_{i j}=0$, and both the OGE and OPE are extremely attractive). In this situation the HHM result with only a few (two) basis functions is simply not converged. If a sufficiently rich basis is employed, the $N$ ground state (as well as some of its excitations) falls down by more than $2000 \mathrm{MeV}$ and additional levels of spin content $S=\frac{1}{2}$ are found below

the $S=\frac{3}{2}$ states in the $J^{P}=\frac{1}{2}^{-}, J^{P}=\frac{3}{2}^{-}$, and $J^{P}=\frac{5}{2}^{-}$bands. The convergence of the energies to the values given in Fig. $1 b$ can be followed in a transparent manner especially in the case of our variational solution of the Schrödinger equation, when gradually increasing the test-function space. ¿From the fully converged results we must then conclude that the

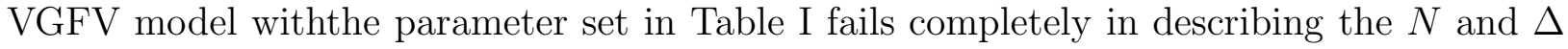
spectra.

\section{B. The model by Dziembowski, Fabre, and Miller}

In the DFM model the effective $Q-Q$ interaction (called "complete Hamiltonian" in Ref. [7]) consists of 


$$
V^{D F M}=V_{O G E}+V_{\chi}+V_{\text {conf }} .
$$

The OGE potential [1] was taken in the usual form but again without spin-orbit and tensor parts - just as in the case of the VGFV model in Eq. (2). Also a linear confinement as in Eq. (5) was used. The potential $V_{\chi}$ relating to broken chiral symmetry was assumed after the suggestion of GBE in Ref. [2]. In the actual calculation, DFM took the central part of the pseudoscalar-octet meson-exchange potential (i.e., for $\pi$ and $\eta$ ) whose radial dependence was parametrized as

$$
V_{\chi}\left(\vec{r}_{i j}\right)=\frac{g_{\pi Q}^{2}}{4 \pi} \frac{1}{12 m_{Q}^{2}} \overrightarrow{\sigma_{i}} \cdot{\overrightarrow{\sigma_{j}}}_{\lambda_{i}}^{F} \cdot{\overrightarrow{\lambda_{j}}}^{F}\left(\mu_{\gamma}^{2} \frac{e^{-\mu_{\gamma} r_{i j}}}{r_{i j}}-\Lambda^{2} \frac{e^{-\Lambda r_{i j}}}{r_{i j}}\right), \quad \gamma=\pi, \eta .
$$

Here, $\vec{\lambda}_{i}{ }^{F}$ are the Gell-Mann flavour matrices, $m_{Q}=336 \mathrm{MeV}$ the constituent quark mass, $\mu_{\gamma}$ the meson masses, and $\Lambda=1 / r_{0}$ a parameter, again from the smearing of the $\delta$-function. Contrary to VGFV, in the DFM model no $\sigma$-exchange was employed. Indeed, the effect of this spin- and flavour-independent scalar interaction can always be incorporated effectively in the confinement potential (5). The fitting parameters of the DFM $Q-Q$ interaction are given in Table [1. The DFM group solved the three- $Q$ system also along the Schrödinger equation with the HHM [8, []. However, they used an even more restricted basis than VGFV, employing only a single state for both positive- as well as negative-parity states and leaving out mixed-symmetry spin-flavour configurations. In case of their "complete Hamiltonian" (i.e. the hybrid model with OGE and one-meson exchange and the parameter set of Table III) they obtained the results shown in Fig. 2a.

We have recalculated the DFM model again by both the stochastic variational and Faddeev methods (as described in the previous section) and found the $N$ and $\Delta$ spectra as shown in Fig. 2b. For adjusting the $N$ ground state to its empirical value of $939 \mathrm{MeV}$ we have to take a constant $V_{0}=-86 \mathrm{MeV}$ in the confinement potential (5). Considerable differences are evident when comparing Figs. 2a and b. Again, most striking is the huge $N-\Delta$ splitting, which turns out to be more than two times larger than in reality. Also the firstexcited levels in the $J^{P}=\frac{1}{2}^{+}, \frac{1}{2}^{-}, \frac{3}{2}^{-}$nucleon bands differ by $40-50 \mathrm{MeV}$; the discrepancies are of a similar 
magnitude in the $J^{P}=\frac{3}{2}^{+}, \frac{5}{2}^{+}$states. The higher excitations in both the positive- and negative-parity bands show unrealistic splittings from the $N$ ground state $(\gtrsim 900 \mathrm{MeV})$. Similarly to the VGFV model additional states appear in the $J^{P}=\frac{1}{2}^{-}$and $\frac{3}{2}^{-}$bands: the

$S=\frac{3}{2}, L=1$ states are found only above a second $S=\frac{1}{2}, L=1$ excitation. From our recalculated spectra it is also evident that in the DFM model the wrong level orderings of positive- and negative-parity excitationspersist. For example, the Roper resonance $N(1440)$ lies above the first negative-parity excitations $N(1535)-N(1520)$; the structure is analogous among the $\Delta$ levels.

Though the DFM potential (6), being less deep than the VGFV potential at short distances (due to the larger value of $r_{0}$ ), is by far easier to compute than the $Q-Q$ interaction of VGFV, the HHM still was not applied on a large enough basis in Ref. [7]. As a result the $N$ level (and some of its excitations) were not converged. If in our stochastic variational calculation we restrict the test-function space in a similar manner as DFM, we can roughly reproduce their results given in Ref. [7]. However, as it becomes evident from the fully converged results in Fig. 2b, the DFM model, with the parameter set in Table [1], grossly fails in describing the $N$ and $\Delta$ spectra.

\section{THE GBE CONSTITUENT QUARK MODEL}

In this Section we discuss a constituent quark model whose $Q-Q$ interaction relies solely on GBE. We like to demonstrate that in this case a unified description of the $N$ and $\Delta$ spectra (and, indeed, also of all strange spectra) can be achieved without the need of advocating a sizeable contribution from OGE. We construct the CQM in a semirelativistic framework [19], i.e. taking the kinetic-energy operator in relativistic form

$$
H_{0}=\sum_{i=1}^{3} \sqrt{{\overrightarrow{p_{i}}}^{2}+m_{i}^{2}}
$$

with $m_{i}$ the masses and $\vec{p}_{i}$ the 3 -momenta of the constituent quarks. It is obvious that this intermediate step towards a fully covariant treatment of the three- $Q$ system (which is still 
beyond scope at present) already constitutes an essential improvement over nonrelativistic approaches. Thereby one can at least avoid several disturbing shortcomings of nonrelativistic CQM. For example, if one calculates the expectation value of the kinetic energy in nonrelativistic CQM, such as the hybrid models of the previous section or the model of Ref. [14], one finds that the ratio of the mean velocity $v$ of a constituent quark to the velocity $c$ of light is $v / c>1$. This clearly indicates that three- $Q$ systems must not be treated in a nonrelativistic manner. By the use of the relativistic kinetic-energy operator in Eq. (8) one avoids such problems a priori and $v / c<1$ is always ensured.

In the GBE constituent quark model, the dynamical part of the Hamiltonian consists of the $Q-Q$ interaction

$$
V=V_{\chi}+V_{\text {conf }}
$$

where $V_{\text {conf }}$ is taken in the usual linear form of Eq. (5) and $V_{\chi}$ is derived from GBE. The latter leads to a spin- and flavour-dependent interaction between constituent quarks $i$ and $j$, whose spin-spin component is manifested by the sum of the pseudoscalar meson-exchange potentials [2, 14]

$$
V_{\chi}^{\text {octet }}\left(\vec{r}_{i j}\right)=\left[\sum_{a=1}^{3} V_{\pi}\left(\vec{r}_{i j}\right) \lambda_{i}^{a} \lambda_{j}^{a}+\sum_{a=4}^{7} V_{K}\left(\vec{r}_{i j}\right) \lambda_{i}^{a} \lambda_{j}^{a}+V_{\eta}\left(\vec{r}_{i j}\right) \lambda_{i}^{8} \lambda_{j}^{8}\right] \vec{\sigma}_{i} \cdot \vec{\sigma}_{j}
$$

and

$$
V_{\chi}^{\text {singlet }}\left(\vec{r}_{i j}\right)=\frac{2}{3} \vec{\sigma}_{i} \cdot \vec{\sigma}_{j} V_{\eta^{\prime}}\left(\vec{r}_{i j}\right),
$$

with $\overrightarrow{\sigma_{i}}$ and $\overrightarrow{\lambda_{i}}$ the quark spin and flavour matrices,respectively. In the simplest derivation, when pseudoscalar or pseudovector couplings are employed in the meson-quark vertices and the boson fields satisfy the linear Klein-Gordon equation, one obtains, in static approximation, the well-known meson-exchange potentials

$$
\begin{gathered}
V_{\gamma}\left(\vec{r}_{i j}\right)=\frac{g_{8}^{2}}{4 \pi} \frac{1}{12 m_{i} m_{j}}\left\{\mu_{\gamma}^{2} \frac{e^{-\mu_{\gamma} r_{i j}}}{r_{i j}}-4 \pi \delta\left(\vec{r}_{i j}\right)\right\}, \quad \gamma=\pi, K, \eta \\
V_{\eta^{\prime}}\left(\vec{r}_{i j}\right)=\frac{g_{0}^{2}}{4 \pi} \frac{1}{12 m_{i} m_{j}}\left\{\mu_{\eta^{\prime}}^{2} \frac{e^{-\mu_{\eta^{\prime}} r_{i j}}}{r_{i j}}-4 \pi \delta\left(\vec{r}_{i j}\right)\right\},
\end{gathered}
$$


with $\mu_{\gamma}$ being the meson masses $\left(\gamma=\pi, K, \eta, \eta^{\prime}\right)$. While the octet of pseudoscalar mesons may be considered as the manifestation of the Goldstone bosons, the singlet $\eta^{\prime}$ is a priori no Goldstone boson due to the axial anomaly. However, in the large- $N_{C}$ limit, the axial anomaly would vanish [20] and the $\eta^{\prime}$ become the ninth Goldstone boson of the spontaneously broken $U(3)_{R} \times U(3)_{L}$ chiral symmetry [21]. Under real conditions with $N_{C}=3$, we may thus consider the contribution from $\eta^{\prime}$ exchange but with a coupling different from the octet mesons (see the discussion later on). The potentials (12) are strictly applicable only for pointlike particles. Since one deals with structured particles (constituent quarks and pseudoscalar mesons) of finite extension, one must smearout the $\delta$-function. The corresponding term turns out to be of crucial importance for the effective $Q-Q$ interaction in baryons: it has a sign appropriate to reproduce the level splittings and it dominates over the Yukawa part towards shorter distances.

There are several choices of smearing the $\delta$-function in Eqs. (12). A reasonable constraint is that the volume integral of the potential should vanish - as it is required for pseudoscalar exchange interactions with finite-mass bosons (mesons); for pure Goldstone bosons of vanishing mass this constraint would not apply, however. In Ref. [14] we have employed the Gaussian-type smearing of the $\delta$-function

$$
4 \pi \delta\left(\vec{r}_{i j}\right) \rightarrow \frac{4}{\sqrt{\pi}} \alpha^{3} e^{-\alpha^{2}\left(r_{i j}-r_{0}\right)^{2}},
$$

which involves two parameters corresponding to the position $\left(r_{0}\right)$ and the breadth $(\alpha)$ of a bell-shaped curve. In this case the volume integral of the chiral potential $V_{\chi}$ does not vanish.

We have found [22] that a Yukawa-type smearing like

$$
4 \pi \delta\left(\vec{r}_{i j}\right) \rightarrow \Lambda_{\gamma}^{2} \frac{e^{-\Lambda_{\gamma} r_{i j}}}{r_{i j}}, \quad \gamma=\pi, K, \eta, \eta^{\prime}
$$

involving the "cut-off" parameters $\Lambda_{\gamma}$, works equally well for the effective $Q-Q$ interaction in baryon spectra. With the $\delta$-function representation (14) the volume integral of $V_{\chi}$ vanishes, thus meeting the requirement mentioned above.

The $\delta$-function representation (14) relates to the finite extension of the meson-quark vertices. If we employ the phenomenological values for the different meson masses $\mu_{\gamma}$, we 
should also allow for a different cut-off parameter $\Lambda_{\gamma}$ corresponding to each meson exchange: with a larger meson mass also $\Lambda$ should increase. Otherwise, the meson-exchange interactions (12) could receive unwarranted contributions from certain meson exchanges (e.g., it could become effectively attractive instead of repulsive or vice versa at short distances). In our attempt to keep the number of free parameters as small as possible, we have avoided fitting each individual cut-off parameter $\Lambda_{\gamma}$, however. Instead, we have succeeded in describing their dependence on the meson mass via the linear scaling prescription

$$
\Lambda_{\gamma}=\Lambda_{0}+\kappa \mu_{\gamma}
$$

which involves only the two parameters $\Lambda_{0}$ and $\kappa$. Their numericalvalues have been determined from a fit to the baryon spectra.

In Eq. (12a) we have foreseen a single octet meson-quark coupling constant $g_{8}^{2} / 4 \pi$. Indeed, in the chiral limit there is only one coupling constant $g_{8}^{2} / 4 \pi$ for all Goldstone bosons. Due to explicit chiral symmetry breaking the coupling constants for $\pi, K$, and $\eta$ may become different. Nevertheless, due to the present lack of firm insight and in order to prevent a proliferation of free parameters, we rely at this stage on a universal coupling constant $g_{8}^{2} / 4 \pi$, which we assume to be equal to the pion-quark coupling constant $g_{\pi Q}^{2} / 4 \pi$. The latter may be deduced from the pion-nucleon coupling using the Goldberger-Treiman relations for both the pion-quark and pion-nucleon vertices:

$$
\frac{g_{8}^{2}}{4 \pi}=\frac{g_{\pi Q}^{2}}{4 \pi}=\left(\frac{g_{Q}^{A}}{g_{N}^{A}}\right)^{2}\left(\frac{m_{Q}}{m_{N}}\right)^{2} \frac{g_{\pi N}^{2}}{4 \pi} .
$$

Here, $g_{Q}^{A}$ and $g_{N}^{A}$ are the quark and nucleon axial coupling constants, $m_{Q}$ and $m_{N}$ the light-quark resp. nucleon masses, and $g_{\pi N}^{2} / 4 \pi=14.2$, the phenomenological $\pi N$ coupling constant [23]. The nucleon and quark axial coupling constants can be related by the ratio

$$
\frac{g_{Q}^{A}}{g_{N}^{A}}=\frac{3}{5}=0.6
$$

If one takes a constituent-quark mass of $m_{Q}=340 \mathrm{MeV}$ (as it is suggested from nucleon magnetic-moment studies) one can deduce the $\pi Q$ coupling constant of the size $g_{\pi Q}^{2} / 4 \pi=$ 
0.67. Thus we fix the octet meson-quark coupling to this value, as it was done already in Ref. [14].

We remark, however, that the $\pi Q$ coupling constant could also assume a value slightly different from 0.67. For instance, if one takes for the $Q$ axial coupling constant $g_{Q}^{A}=1$ (as it should be in the large- $N_{C}$ limit [24]) and employs for the $N$ axial coupling constant its phenomenological value [23] $g_{N}^{A}=1.25$, then one obtains the ratio

$$
\frac{g_{Q}^{A}}{g_{N}^{A}}=0.8 .
$$

Thus one would end up with a pion-quark coupling constant $g_{\pi Q}^{2} / 4 \pi=1.19$. If one took into account that $1 / N_{C}$ corrections would reduce the $Q$ axial coupling constant to $g_{Q}^{A}=0.87$ 25], then

$$
\frac{g_{Q}^{A}}{g_{N}^{A}}=0.7,
$$

and consequently $g_{\pi Q} / 4 \pi=0.91$. Therefore we may ultimately expect the $\pi Q$ coupling constant within the interval $0.67 \lesssim g_{\pi Q}^{2} / 4 \pi \lesssim 1.19$ and this is even dependent on the value of the constituent-quark mass adopted. Still, at this stage, we need not vary the size of the octet meson-quark coupling constant and simply fix it to the "canonical" value of $\approx 0.67$.

Due to the special character of the singlet $\eta^{\prime}$ meson (see the discussion above), its coupling was allowed to deviate from the universal octet coupling. In the actual fit, the ratio $\left(g_{0} / g_{8}\right)^{2}$ turns out larger than 1. However, this is in line with results deduced in Refs. [26,27, from the Gottfried sum-rule violation, the $\bar{u} / \bar{d}$ ratio, as well as the spin content of the nucleon.

In the semirelativistic GBE model we have a confinement strength (of the linear confining potential, Eq. (5)) of the order of $C \approx 2.3 \mathrm{fm}^{-2}$. We note that this value appears to be quite realistic, as it is consistent both with Regge slopes and also the string tension extracted in lattice QCD [28]. The size of the confinement strength required in our semirelativistic GBE model represents another comfortable improvement over nonrelativistic CQM where a much smaller value of $C$ must be chosen (see the examples of the previous section or Refs. [14, 22]).

For the whole $Q-Q$ potential in Eq. (9) our model now involves a total of five free parameters whose numerical values are given in Table III. We remark, however, that this is 
only one fit out of a possible set of others that lead to a similar quality in the description of the baryon spectra. For instance, we have found that one could increase the octet coupling constant $g_{8}^{2} / 4 \pi$ and compensate the change by readjusting the other parameters, particularly the cut-offs $\Lambda_{\gamma}$.Similarly, the ratio $\left(g_{0} / g_{8}\right)^{2}$ could be lowered, and even the constituent-quark masses may be chosen differently. Retuning the other parameters, including the confinement strength $C$, one can within certain limits always obtain a fit of comparable quality.

The GBE model with the semirelativistic three- $Q$ Hamiltonian relying on Eqs. (8) and (9) was solved along the stochastical variational method [1] in momentum space. The convergence was carefully tested, especially with respect to the $N$ ground state and the "dangerous" levels in the excitation spectra.

In Fig. 3 we present the ground-state and excitation levels of the $N$ and $\Delta$ spectra as produced by the semirelativistic GBE constituent quark model with the $Q$ - $Q$ potential from Eqs. (91)-(12), the Yukawa-type representation (14) of the $\delta$-function and the parameter set given in Table [1]. The light- $Q$ masses were taken as $m_{u}=m_{d}=m_{Q}=340 \mathrm{MeV}$, and for the meson masses $\mu_{\gamma}$ the phenomenological values were employed.

Tensor force effects are not yet included in these results, as is evident from the absence of any fine-structure splittings in the theoretical $L S$-multiplets. It is clear, however, that tensor forces can at most play a subordinate role in the $N$ and $\Delta$ spectra. First of all, this follows from the smallness of the level splittings of corresponding $L S$-multiplets in the experimental spectra. Second, from a first numerical estimation of the influence of the pseudoscalar meson-exchange tensor forces prevailing in the GBE model we have found only small effects on the baryon states.

¿From the results of Fig. 3 it becomes evident that within the GBE constituent quark model a unified description of both the $N$ and $\Delta$ spectra is achieved in good agreement with phenomenology. Practically all levels are found in their "experimental boxes" and specifically the orderings of positive- and negative-parity excitations are correct. 


\section{DISCUSSION}

As already outlined before [2, 14], the successes of the GBE constituent quark model are due to the specific spin-flavour symmetry inherent in the chiral potential $V_{\chi}$ in Eqs. (10) and (11). It produces a level structure well adjusted to the experimental data. This is not only true in the $N$ and $\Delta$ cases but holds also for strange baryons [22,29]. With the possible exception of the empirically large $\Lambda(1405)-\Lambda(1520)$ splitting, which remains unexplained at this stage, all octet/decuplet states together with their excitations can be described in a unified manner.

¿From the results of the GBE constituent quark model exemplified in the previoussection we also learn that no (additional) OGE is needed for the reproduction of the baryon spectra, contrary to the claims made for the hybrid models, e.g., in Refs. [6] and [7]. Still, one could accommodate a certain contribution from OGE but only with a rather small effect from the colour-magnetic interaction. As soon as the colour-magnetic forces become strong, like it is the case in the hybrid models discussed in Sec. II, one faces the traditional difficulties in the light-baryon spectra. If in a meson-exchange plus OGE interaction with a sizeable contribution from the colour-magnetic interaction the $N-\Delta$ splitting (to which both types of exchanges contribute in the same manner) is described correctly, the correct ordering of positive- and negative-parity levels is not achieved, because the meson-exchange contribution turns out simply too weak. Only a bigger influence from meson (pion) exchange would allow the first positive-parity excitations in the $N$ spectrum to fall below the first negative-parity excitations. If, on the other hand, the meson exchange is made strong enough to achieve this level inversion (as demanded by phenomenology), the colour-magneticcontribution must remain very small, practically negligible, in order not to spoil a correct $N-\Delta$ splitting. Thus it is quite naturally suggested by the experimental level structure that OGE can at most play a subordinate role in CQM for light baryons.

Finally we note that a nonrelativistic treatment of three- $Q$ systems cannot really work. Evidently, light (and strange) three- $Q$ systems already bear large relativistic effects of kine- 
matical origin. They call at least for the kinetic energy part of the full Hamiltonian to be employed in relativistic form, as was done for the GBE model in Sec. III. Otherwise the lack of taking into account (kinematical) relativistic effects explicitly will be compensated within the $Q-Q$ potential parameters in the fit to the baryon spectra. Consequently, in this procedure the model parameters will assume rather unrealistic values, e.g., the confinement strength would turn out to be only half of the one resulting for the string tension from lattice QCD and the one needed to fit Regge slopes. As a result, any nonrelativistic CQM can at most be considered as a parametrization of the baryon energy levels, rather than as a dynamical model for light three- $Q$ systems. Certainly it will not prove acceptable for future applications such as the description of electromagnetic form factors, hadronic decays, and other dynamical observables that are determined by the behaviour of the baryon wave functions and are generally much influenced by $Q-Q$ potential parameters.

\section{ACKNOWLEDGMENT}

The authors acknowledge clarifying correspondence with P. González, A. Valcarce, and V. Vento as well as discussions with G.A. Miller. They have also profited from valuable discussions with D.O. Riska as well as B.L.G. Bakker, V.B. Mandelzweig, and M. Rosina. The

work was partially supported by the Austrian-Hungarian Scientific-Technical Cooperation, within project A23 resp. 17/95, and by the Paul-Urban Foundation. 


\section{REFERENCES}

[1] A. de Rújula, H. Georgi, and S. L. Glashow, Phys. Rev. D 12, 147 (1975).

[2] L. Ya. Glozman and D. O. Riska, Phys. Rep. 268, 263 (1996).

[3] L. Ya. Glozman, in: Perturbative and Nonperturbative Aspects of Quantum Field Theory (Lecture Notes in Physics, vol. 479). Eds.: H. Latal and W. Schweiger. Springer, Berlin, 1997.

[4] I. T. Obukhovsky and A. M. Kusainov, Phys. Lett. B 238, 142 (1990).

[5] Z. Zhang, A. Faessler, U. Straub, and L. Ya. Glozman, Nucl. Phys. A578, 573 (1994);

Y. Fujiwara, C. Nakamoto, and Y. Suzuki, Phys. Rev. Lett. 76, 2242 (1996).

[6] A. Valcarce, P. González, F. Fernández, and V. Vento, Phys. Lett. B 367, 35 (1996).

[7] Z. Dziembowski, M. Fabre de la Ripelle, and G. A. Miller, Phys. Rev. C 53, R2038 (1996).

[8] J. L. Ballot and M. Fabre de la Ripelle, Ann Phys. (N.Y.) 127, 62 (1980).

[9] M. Fabre de la Ripelle, H. Fiedeldey, and S. A. Sofianos, Few-Body Systems 2, 493 (1987).

[10] Z. Papp and W. Plessas, Phys. Rev. C 54, 50 (1996).

[11] K. Varga and Y. Suzuki, Phys. Rev. C 52, 2885 (1995).

[12] B. Silvestre-Brac and C. Gignoux, Phys. Rev. D 32, 743 (1985).

[13] B. Desplanques, C. Gignoux, B. Silvestre-Brac, P. González, J. Navarro, and S. Noguera, Z. Phys. A 343, 331 (1992); B. Silvestre-Brac, Few-Body Systems 20, 1 (1996).

[14] L. Ya. Glozman, Z. Papp, and W. Plessas, Phys. Lett. B 381, 311 (1996).

[15] K. Varga, Y. Ohbayasi, and Y. Suzuki, Phys. Lett. B 396, 1 (1997). 
[16] P. González, private communication.

[17] A. Valcarce, A. Buchmann, F. Fernández, and A. Faessler, Phys. Rev. C 50, 2246 (1994).

[18] A. Valcarce, private communication.

[19] J. Carlson, J. Kogut, and V. R. Pandharipande, Phys. Rev. D 27, 233 (1983).

[20] E. Witten, Nucl. Phys. B 156, 269 (1979).

[21] S. Coleman, E. Witten, Phys. Rev. Lett. 45, 100 (1980).

[22] L. Ya. Glozman, Z. Papp, W. Plessas, K. Varga, and R. F. Wagenbrunn, in: Proceedings

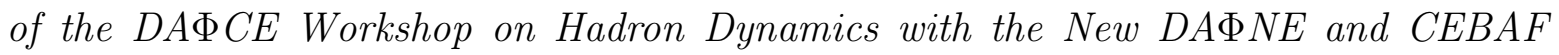
Facilities, Frascati, 1996; to appear in Nucl. Phys. A.

[23] T. Ericson and W. Weise, Pions in Nuclei. Clarendon Press, Oxford, 1988.

[24] S. Weinberg, Phys. Rev. Lett. 65, 1181 (1990).

[25] S. Weinberg, Phys. Rev. Lett. 67, 3473 (1991).

[26] T. P. Cheng and L.-F. Li, Phys. Rev. Lett. 74, 2872 (1995).

[27] T. P. Cheng and L.-F. Li, Phys. Lett. B 366, 365 (1996).

[28] M. Creutz, Quarks, Gluons and Lattices. Cambridge Univ. Press, Cambridge, 1983.

[29] L. Ya. Glozman, Z. Papp, W. Plessas, K. Varga, and R. F. Wagenbrunn, in preparation. 


\section{FIGURES}
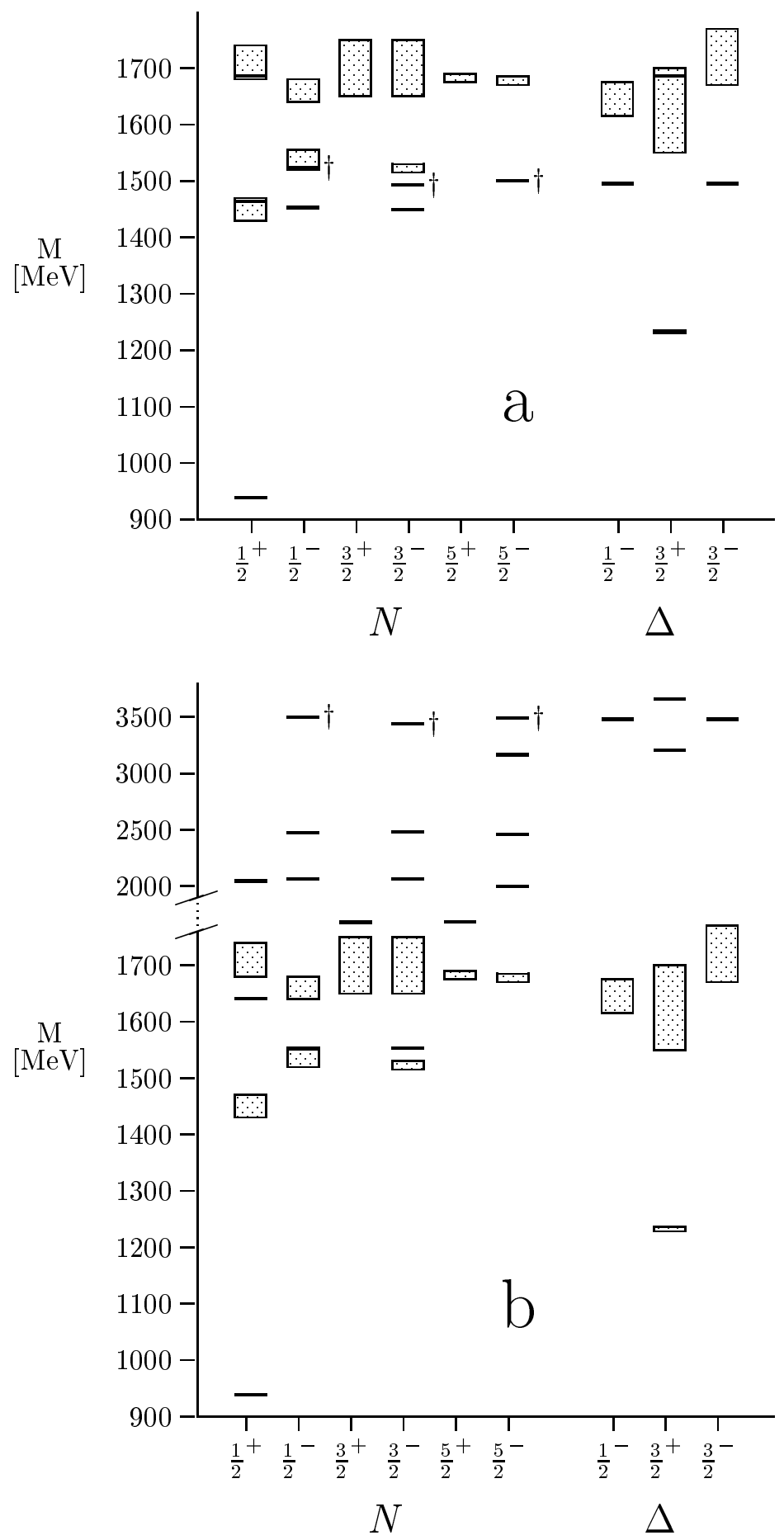

FIG. 1. $N$ and $\Delta$ spectra in case of the model VGFV: a) as given in Ref. [6], b) recalculated. In both cases the nucleon mass is "normalized" to its phenomenological value of $939 \mathrm{MeV}$. In the recalculated spectra the $N-\Delta$ mass difference is $2270 \mathrm{MeV}$. The negative-parity states marked by a dagger correspond to the spin content of predominantly $S=\frac{3}{2}$. 

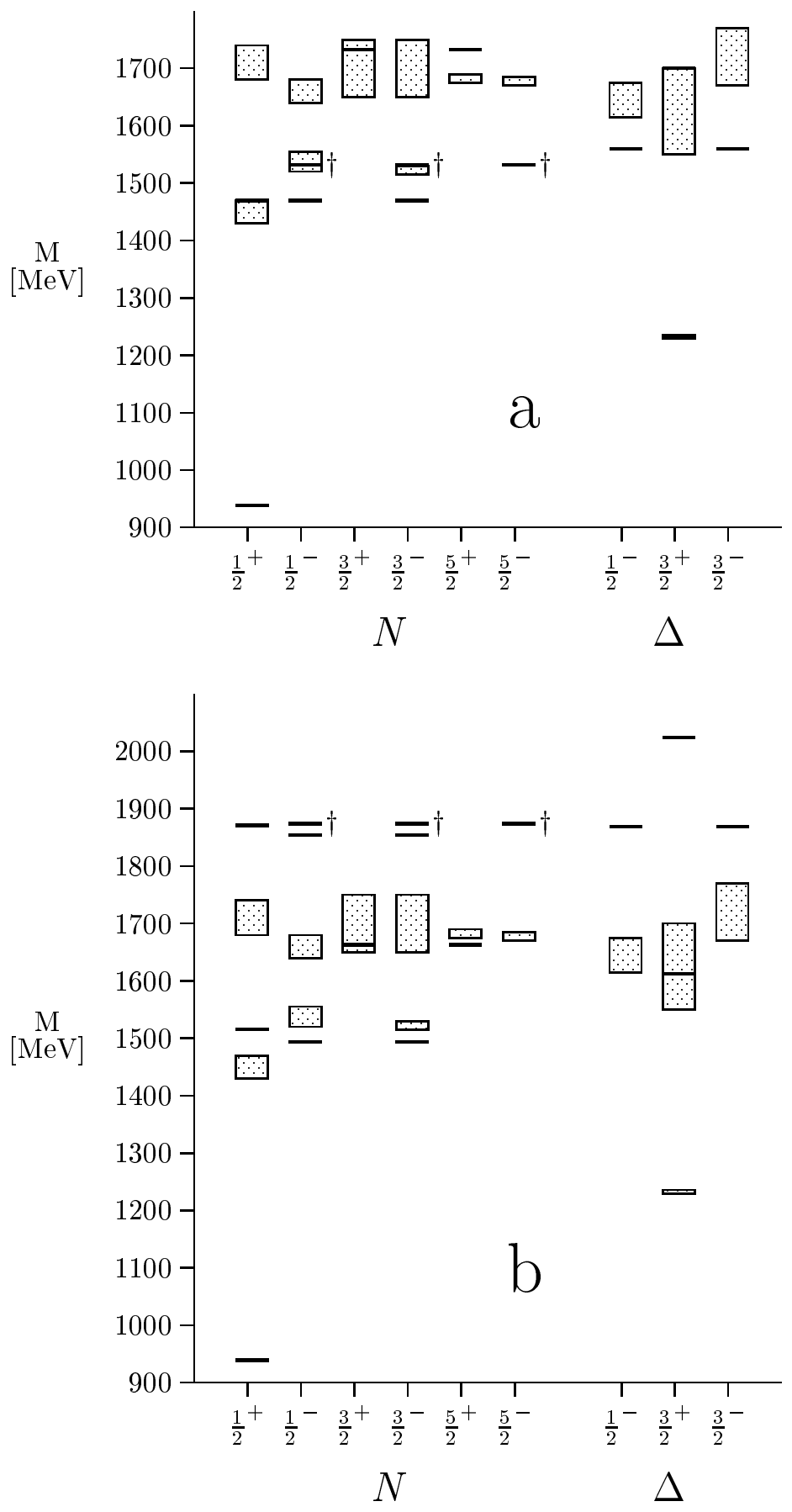

FIG. 2. $N$ and $\Delta$ spectra in case of the model DFM: a) as given in Ref. [7], b) recalculated. In both cases the nucleon mass is "normalized" to its phenomenological value of $939 \mathrm{MeV}$. In the recalculated spectra the $N-\Delta$ mass difference is $670 \mathrm{MeV}$. The negative-parity states marked by a dagger correspond to the spin content $S=\frac{3}{2}$. 


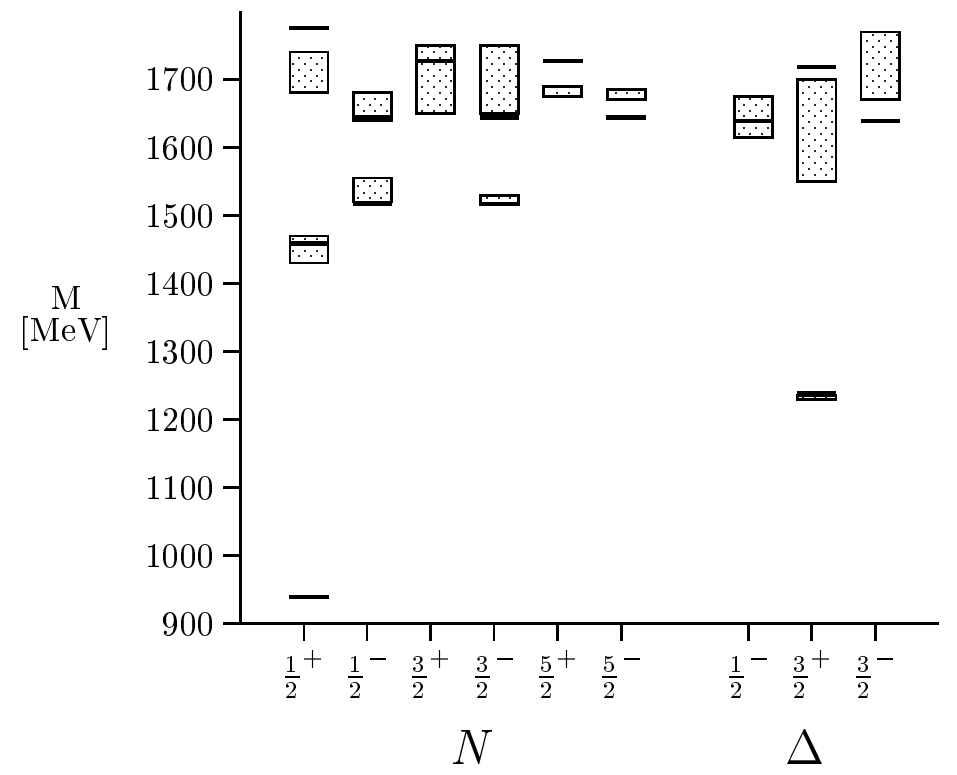

FIG. 3. Energy levels of the 14 lowest light-baryon states with total angular momentum and parity $J^{P}$ in case of the semirelativistic GBE constituent quark model with parameters as given in Table III. The shadowed boxes represent the experimental values with their uncertainties; the $N$ and $\Delta$ ground-state levels coincide with the experimental values. 


\section{TABLES}

TABLE I. Numerical values of fitting parameters of the VGFV $Q-Q$ interaction. Three of the parameters, namely, $\alpha_{s}, g_{\pi Q}^{2} / 4 \pi$, and $\Lambda$ were taken from the hybrid $N-N$ interaction model in Ref. 17.

\begin{tabular}{lccccc}
\hline \hline$\alpha_{s}$ & $g_{\pi Q}^{2} / 4 \pi$ & $C\left[\mathrm{fm}^{-2}\right]$ & $V_{0}[\mathrm{MeV}]$ & $r_{0}[\mathrm{fm}]$ & $\Lambda\left[\mathrm{fm}^{-1}\right]$ \\
\hline 0.485 & 0.545 & $0.980^{\mathrm{a}}$ & $+488^{\mathrm{b}}$ & 0.0367 & 4.2 \\
\hline \hline
\end{tabular}

aThere is a misprint in the value of the confinement strength in Ref. [6]. The correct value given here was communicated to us by the Salamanca-Valencia group 18].

${ }^{\mathrm{b}}$ The constant $V_{0}$ in the confinement potential, whose value is not quoted in Ref. [6], must be taken of this magnitude in order to adjust the nucleon ground state in our (converged) calculation.

TABLE II. Numerical values of fitting parameters of the DFM $Q-Q$ interaction. The cut-off parameter $\Lambda$ in the meson-exchange potentials (7) was chosen to be the same as in the OGE part, i.e. $\Lambda=1 / r_{0}$.

\begin{tabular}{lcccc}
\hline \hline$\alpha_{s}$ & $g_{\pi Q}^{2} / 4 \pi$ & $C\left[\mathrm{fm}^{-2}\right]$ & $V_{0}[\mathrm{MeV}]$ & $r_{0}[\mathrm{fm}]$ \\
\hline 0.35 & 1.15 & 1.014 & $-86^{\mathrm{a}}$ & 0.238 \\
\hline \hline
\end{tabular}

aThe constant $V_{0}$ in the confinement potential,whose value is not quoted in Ref. [7], must be taken of this magnitude in order to adjust the nucleon ground state in our (converged) calculation.

TABLE III. Numerical values of fitting parameters of the semirelativistic GBE $Q$ - $Q$ interaction. The octet meson-quark coupling constant was fixed to $g_{8}^{2} / 4 \pi=0.67$.

\begin{tabular}{lcccc}
\hline \hline$\left(g_{0} / g_{8}\right)^{2}$ & $C\left[\mathrm{fm}^{-2}\right]$ & $V_{0}[\mathrm{MeV}]$ & $\Lambda_{0}\left[\mathrm{fm}^{-1}\right]$ & $\kappa$ \\
\hline 1.34 & 2.33 & -416 & 2.87 & 0.81 \\
\hline \hline
\end{tabular}

